\title{
Development and validation of a multivariable risk prediction model for head and neck cancer using the UK Biobank
}

\author{
CAROLINE ELIZABETH McCARTHY ${ }^{1,2}$, LAURA JAYNE BONNET ${ }^{3}$, \\ MICHAEL WILLIAMS MARCUS ${ }^{2}$ and JOHN K. FIELD ${ }^{1,2}$ \\ ${ }^{1}$ Department of Oral Medicine, Liverpool University Dental Hospital, Pembroke Place, \\ Liverpool, Merseyside L3 5PS; ${ }^{2}$ Department of Molecular and Clinical Cancer Medicine, \\ Institute of Translational Medicine, University of Liverpool, Liverpool, Merseyside L7 8TX; ${ }^{3}$ Department of Biostatistics, \\ Institute of Translational Medicine, University of Liverpool, Liverpool, Merseyside L69 3GL, UK
}

Received March 18, 2020; Accepted July 7, 2020

DOI: $10.3892 /$ ijo.2020.5123

\begin{abstract}
Head and neck cancer (HNC) is the eighth most common cancer in the UK, with over 12,000 new cases every year. The incidence of HNC is predicted to increase by $33 \%$ by 2035 . Risk modelling produces personalised risk estimates for specific diseases, which can be used to inform education, screening programmes and recruitment to clinical trials. The present study describes the development and validation of the first risk prediction model for absolute risk of $\mathrm{HNC}$, using a nested case-control study within the UK Biobank dataset. The UK Biobank recruited 502,647 individuals aged 40-69 years from around the UK. In total, 859 cases of HNC were identified, with 253 incident cases (individuals who developed HNC in the 7 years following recruitment to the UK Biobank study). Logistic regression was used to develop the model, then the model performance was validated using a cohort from the North West of England. Overall, increasing age, male sex, positive history of smoking and alcohol consumption and higher levels of material deprivation were significantly associated with a higher risk of HNC. Consuming at least five portions of fruit and vegetables per day, exercising at least once per week and higher BMI offered a protective effect against HNC. The C-statistic was 0.69 [95\% confidence interval (CI), 0.66-0.71] and the model displayed good calibration. Upon external validation, the $\mathrm{C}$-statistic was 0.64 (95\% CI, 0.60-0.68) with
\end{abstract}

Correspondence to: Dr Caroline Elizabeth McCarthy, Department of Molecular and Clinical Cancer Medicine, Institute of Translational Medicine, University of Liverpool, The William Henry Duncan Building, 6 West Derby Street, Liverpool, Merseyside L7 8TX, UK E-mail: caroline.mccarthy@liverpool.ac.uk

Abbreviations: HNC, head and neck cancer; BMI, body mass index; NHS, National Health Service

Key words: head and neck cancer, oral cancer, mouth cancer, oropharyngeal cancer, risk prediction, risk modelling, risk factors, personalised risk prediction, cancer risk prediction, epidemiology reasonable calibration. The model developed and validated in the present study allows calculation of a personalised risk estimate for HNC. This could be used to guide clinicians when counselling individuals on risk behaviour, and there is potential for such models to inform recruitment to screening trials.

\section{Introduction}

There were 12,000 cases of head and neck cancer (HNC) in the UK in 2015 and its incidence is predicted to continue to increase by 33\% between 2014 and 2035 (1). The burden of disease is unequal within the UK, with the highest incidence being in areas of social deprivation $(1,2)$. Early detection of $\mathrm{HNC}$ reduces morbidity and mortality rates and decreases the economic burden placed on the NHS (3). Management is less invasive when the disease is treated at earlier stages and results in improved quality of life for patients (4). Patient's displaying symptoms of oral cancer, who are ultimately diagnosed with the disease, are known to delay seeking healthcare advice for $>1$ month in the majority of cases (56.7\%), with $10 \%$ waiting over one year (5).

Risk prediction models offer an opportunity to enhance patient care for HNC. The results from personalised risk calculators could be used during patient consultations to communicate levels of risk to individual patients and assist in counselling on risk behaviours (prevention), whilst also providing an opportunity to enhance patient awareness of the signs and symptoms of disease (6), with the aim of promoting early detection (7). There is also potential for a risk calculator to be used in clinical trial design to enable recruitment of sub-groups of patients with the highest risk of disease. Risk models have been successfully developed and implemented in this context in other cancer types, such as breast (8), colorectal (9) and lung cancer (10). Use of risk prediction models to select individuals at high-risk of lung cancer screening has been recommended, as this improves cost-effectiveness and reduces the risk of false-positive diagnoses $(11,12)$. Therefore, the construction of such risk prediction models for HNC may be useful in the selection of high-risk individuals who would benefit the most from screening (12).

The aim of the present study was to develop and validate the first risk prediction model for HNC in the UK population, 
to the best of our knowledge, to estimate absolute risk of developing the condition.

\section{Materials and methods}

Transparent reporting of a multivariable prediction model for individual prognosis or diagnosis (TRIPOD) guidelines for development, validation and reporting have been followed (13).

Data source. The risk prediction model was developed using a nested case-control study within the UK Biobank dataset (14). The UK Biobank recruited over 500,000 individuals aged 40-69 years between 2006 and 2010, in 35 assessment centres. Participants first completed the consent process via a touch-screen electronic system, allowing for direct data entry. A touch-screen questionnaire was used to collect the majority of data. The individuals were asked questions concerning the following categories: Sociodemographic factors; smoking and alcohol consumption; sexual history, family history of major diseases (including diabetes, some cancer types and cardiovascular disease) and early life factors (for example, infant feeding and maternal smoking); general health and disability; environmental factors; dietary habits; physical activity and psychological and cognitive state. A subsequent computer-assisted personal interview was conducted by trained assessors based on 'screening' questions asked as part of the touch-screen questionnaire. For example, patients who indicated they had a particular medical condition would be asked follow-up questions on this during the interview. In addition, the individuals agreed to be followed up via online questionnaires and via links to national data registries (15). Baseline physical measurements were also recorded, including blood pressure, weight, height, waist and hip circumference, bio-impedance (body-fat), hand-grip strength and bone densitometry were measured.

Outcome. Cases were defined as patients with a diagnosis of $\mathrm{HNC}$, as defined by International Statistical Classification of Diseases and Related Health Problems-10 codes C00-14 and C30-31 (16), and this was used as the outcome measure. Laryngeal cancer was excluded when building this model, as screening for oral cancers and laryngeal cancers requires different expertise and laryngeal cancer would not be visible during routine oral examination. Controls included all participants of the UK Biobank study who did not have a diagnosis of HNC recorded in the Cancer Registries data in September 2016 (15). The UK Biobank is linked to the Cancer Registries and is updated to include newly diagnosed disease after each study period. Some patients had a diagnosis of HNC prior to recruitment to the UK Biobank study and others developed the disease during or after the study period, and only incident cases of HNC (individuals who developed HNC in the 7 years following recruitment to the UK Biobank study) were included when developing the present risk model.

Predictors. The UK Biobank dataset contains 7,800 separate data entries (variables) for each individual. These were reduced to a list of $\sim 250$ candidate predictors for consideration for the present risk model, based on known clinical associations with HNC (17). Data regarding age, sex, smoking, alcohol, diet and exercise, body mass index (BMI), medical history and social demographics were selected for further analysis, based on clinical relevance from literature review (18-20) and feedback from patients. Human papillomavirus (HPV) infection is recognised as a risk factor for oropharyngeal cancers (21), but HPV status was not available in the UK Biobank dataset. However, having $\geq$ six lifetime sexual partners is known to be associated with an increased risk of HPV-associated oropharyngeal cancer $(22,23)$, therefore this was considered for inclusion as a surrogate marker of HPV infection for the present model.

Validation dataset. Validation of risk prediction models is of paramount importance to determine their generalisability in different populations. Models should be validated in external datasets to confirm their generalisability and predictive accuracy, before they are used in clinical practice (24-26). The North West of England is known to have a higher incidence of head and neck cancer compared with other parts of the UK $(2,27)$. For this reason, the cohort dataset was split geographically, into development and validation sets, to test the model's performance in a cohort known to have a higher risk of HNC compared with the cohort used to develop the model $(25,28)$. All participants recruited at assessment centres in the North West of England, including Manchester, Liverpool, Bury and Stockport, were included in the validation dataset. The remaining participants were retained in the model development dataset. The development dataset contained 702 cases of head and neck cancer, of which 199 were incident cases and 423,050 controls. The validation dataset contained 78,895 individuals with 157 cases of $\mathrm{HNC}$, of which 54 are incident cases.

\section{Statistical analysis}

Data handling. Statistical analysis was completed using Stata version 13 statistical software (StataCorp LLC). Continuous variables, such as age, were modelled as continuous to prevent biological implausibility and inefficient use of data (29). However, to facilitate clinical interpretation, the Townsend Deprivation Index (TDI) variable was categorised into recognised quintiles $1-5$ to allow for more meaningful analysis and interpretation of results, with 1 representing least deprived (30). Fruit and vegetable intake was combined and categorised into ' $<$ five per day' and ' $\geq$ five per day', in line with current NHS guidelines that everyone should eat at least five portions (400 g) of fruit and vegetables every day (31). Exercise was also categorised into 'no exercise' or 'moderate exercise for at least $10 \mathrm{~min}, 1-4$ days per week' and 'moderate exercise on 5 or more days of the week', in line with current NHS guidelines on exercise (32).

Missing data. A previous study has shown that irrespective of the missing data mechanism, complete case analysis does not pose a major threat to statistical power in datasets with small amounts of missing data (33). In the present study, missing data was minimal $[<1 \%$ for all variables except exercise (missing data $=5.9 \%$ )], therefore, complete case analysis was used. Total numbers for each variable are shown in Table I. The final model was developed based on 232 incident cases of HNC and 396,947 controls (events per variable=18). 


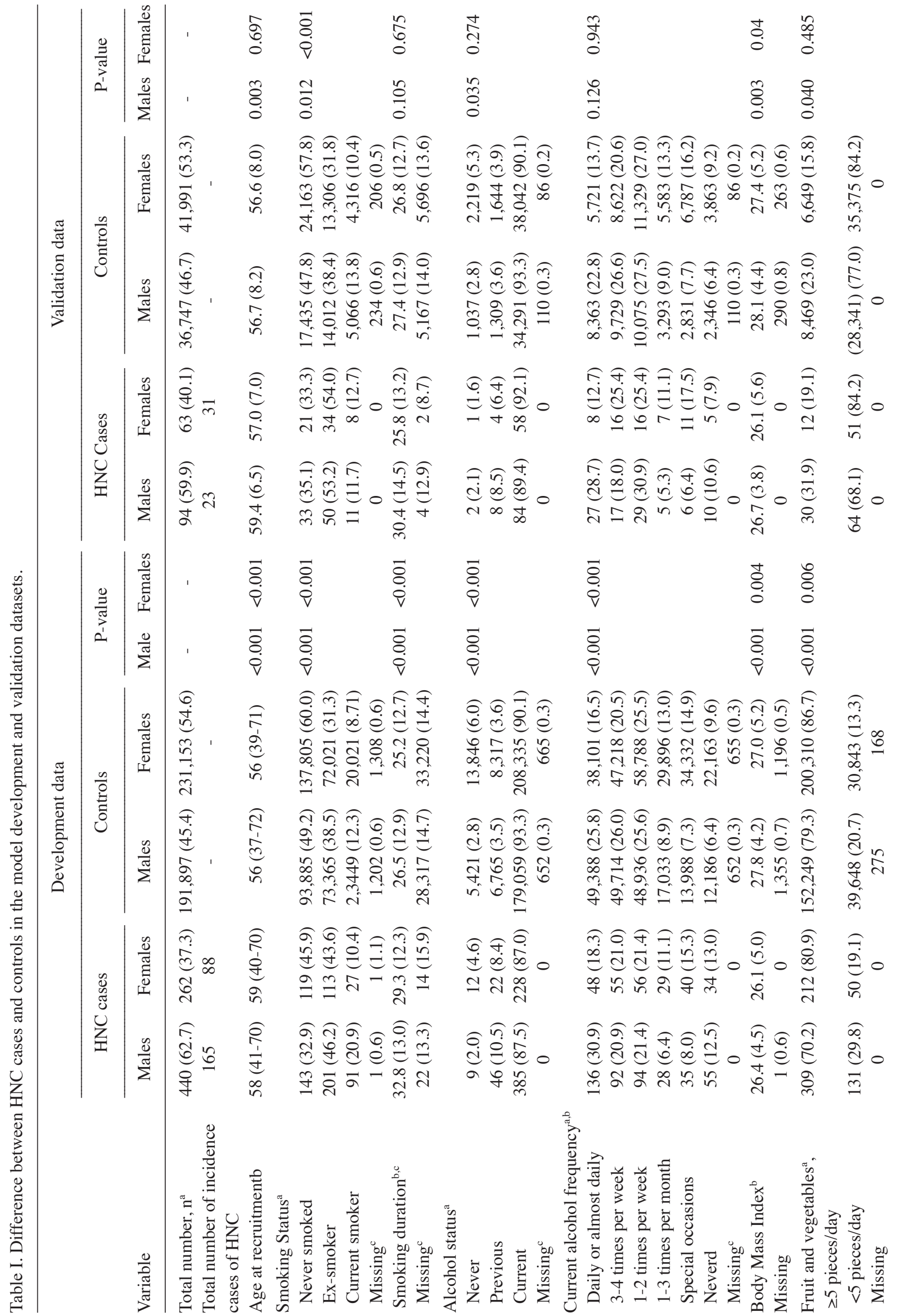




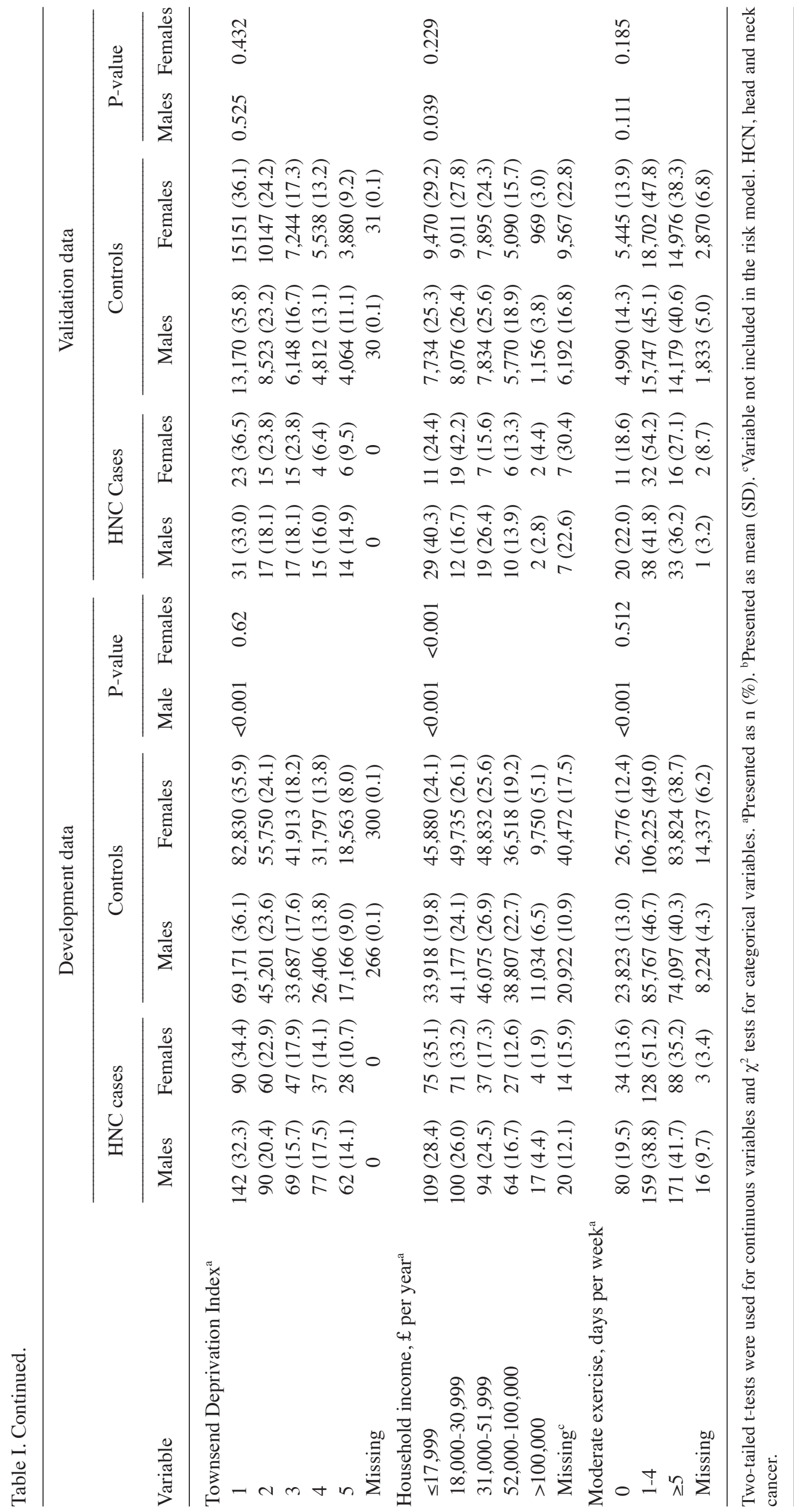


Table II. Univariate analysis of head and neck cancer risk in the UK Biobank.

\begin{tabular}{|c|c|c|c|}
\hline Variable & Odds ratio & $95 \%$ confidence interval & P-value \\
\hline Age, years & 1.04 & $1.02-1.05$ & $<0.001$ \\
\hline \multicolumn{4}{|l|}{ Sex } \\
\hline Female & 1 & & \\
\hline Male & 2.26 & $1.74-2.92$ & $<0.001$ \\
\hline \multicolumn{4}{|l|}{ Smoking status } \\
\hline Never smoked & 1 & & \\
\hline Ex-smoker & 1.95 & $1.46-2.60$ & $<0.001$ \\
\hline Current Smoker & 4.06 & $2.93-5.61$ & $<0.001$ \\
\hline \multicolumn{4}{|l|}{ Alcohol Status } \\
\hline Never drinker & 1 & & \\
\hline Previous drinker & 5.31 & $2.18-13.0$ & $<0.001$ \\
\hline Current drinker & 1.84 & $0.82-4.13$ & 0.141 \\
\hline \multicolumn{4}{|l|}{ Alcohol Frequency } \\
\hline Daily & 1 & & \\
\hline 3-4 times per week & 0.73 & $0.51-1.04$ & 0.081 \\
\hline 1-2 times per week & 0.82 & $0.59-1.15$ & 0.267 \\
\hline 1-3 times per month & 0.42 & $0.24-0.74$ & 0.003 \\
\hline Special occasions & 0.58 & $0.35-0.94$ & 0.028 \\
\hline Never & 1.19 & $0.78-1.83$ & 0.411 \\
\hline Body Mass Index & 0.98 & $0.95-1.01$ & 0.163 \\
\hline Fruit and vegetable intake, $\geq 5$ pieces/day & 0.58 & $0.44-0.77$ & $<0.001$ \\
\hline \multicolumn{4}{|l|}{ Townsend Deprivation Index } \\
\hline 1 & 1 & & \\
\hline 2 & 1.29 & $0.90-1.82$ & 0.25 \\
\hline 3 & 1.21 & $0.82-1.79$ & 0.192 \\
\hline 4 & 2.04 & $1.42-2.91$ & $<0.001$ \\
\hline 5 & 2.06 & $1.36-3.12$ & 0.001 \\
\hline \multicolumn{4}{|l|}{ Household Income, $£$ per year } \\
\hline$\leq 17,999$ & 1 & & \\
\hline $18,000-30,999$ & 0.95 & $0.67-1.35$ & 0.78 \\
\hline $31,000-51,999$ & 0.66 & $0.45-0.97$ & 0.032 \\
\hline $52,000-100,000$ & 0.65 & $0.43-0.99$ & 0.042 \\
\hline$>100,000$ & 0.64 & $0.33-1.25$ & 0.192 \\
\hline \multicolumn{4}{|l|}{ Moderate exercise, number days/week } \\
\hline 0 & 1 & & \\
\hline $1-4$ & 0.6 & $0.42-0.85$ & 0.005 \\
\hline$\geq 5$ & 0.66 & $0.46-0.94$ & 0.022 \\
\hline
\end{tabular}

Model development. Two-tailed unpaired t-tests were used to describe differences between male and female cases and controls for each continuous, clinically relevant variable. $\chi^{2}$ tests were used to test associations between categorical variables (Tables I and SI). $\mathrm{P}<0.05$ was considered to indicate a statistically significant difference.

Each variable of interest was then tested for an (unadjusted) association with the outcome, diagnosis of head and neck cancer, using univariate logistic regression analysis (Table II). This step was performed to detect associations, not to aid in variable selection (34). Final predictors were selected based on clinical significance with regards to published literature, as aforementioned, and following discussion with a local Patient and Public Involvement group (the Liverpool Oral Medicine Patient Research Forum). The patient group were asked to identify questions they would be willing to answer to inform predictor choice, in the context of generating a risk estimate for developing HNC. Smoking status was included as an indicator of current or previous smoking experience. Alcohol status was also used as a reflection of lifetime exposure to alcohol, rather than current alcohol frequency, which does not consider previous intake of alcohol. Type of alcohol was also explored but ultimately excluded from the final model as this cannot be used as a measure of lifetime exposure. 
Table III. Multivariable model for head and neck cancer risk in the UK Biobank.

\begin{tabular}{|c|c|c|c|c|}
\hline \multirow{2}{*}{$\begin{array}{l}\text { Variable } \\
\text { Age, years }\end{array}$} & \multirow{2}{*}{$\begin{array}{c}\text { Odds ratio } \\
1.04\end{array}$} & \multirow{2}{*}{$\begin{array}{c}\text { P-value } \\
<0.001\end{array}$} & \multicolumn{2}{|c|}{$\begin{array}{c}95 \% \text { confidence } \\
\text { interval }\end{array}$} \\
\hline & & & 1.02 & 1.05 \\
\hline \multicolumn{5}{|l|}{ Sex } \\
\hline Female & 1 & & & \\
\hline Male & 1.81 & $<0.001$ & 1.38 & 2.38 \\
\hline \multicolumn{5}{|l|}{ Smoking status } \\
\hline Never & 1 & & & \\
\hline Previous & 1.59 & 0.003 & 1.17 & 2.15 \\
\hline Current & 3.1 & $<0.001$ & 2.17 & 4.4 \\
\hline \multicolumn{5}{|c|}{ Townsend deprivation index } \\
\hline 1 & 1 & & & \\
\hline 2 & 1.3 & 0.177 & 0.9 & 1.81 \\
\hline 3 & 1.14 & 0.499 & 0.77 & 1.7 \\
\hline 4 & 1.81 & 0.001 & 1.25 & 2.63 \\
\hline 5 & 1.66 & 0.02 & 1.08 & 2.56 \\
\hline Body Mass Index & 0.96 & 0.011 & 0.93 & 0.99 \\
\hline \multicolumn{5}{|c|}{ Alcohol consumption } \\
\hline Never & 1 & & & \\
\hline Previous & 3.26 & 0.01 & 1.32 & 8.04 \\
\hline Current & 1.42 & 0.406 & 0.62 & 3.21 \\
\hline \multicolumn{5}{|c|}{ Moderate Exercise, number of days per week } \\
\hline 0 & 1 & & & \\
\hline $1-4$ & 0.68 & 0.04 & 0.47 & 0.98 \\
\hline$\geq 5$ & 0.66 & 0.03 & 0.46 & 0.95 \\
\hline \multicolumn{5}{|c|}{ Fruit and vegetable intake per day } \\
\hline$<5$ & 1 & & & \\
\hline$\geq 5$ & 0.71 & 0.031 & 0.53 & 0.97 \\
\hline
\end{tabular}

Model Intercept Coefficient -9.54 (95\% confidence interval, $-11.2--7.88 ; \mathrm{P}<0.001$ ). Based on 397,179 observations ( $\mathrm{n}=232$ cases and $\mathrm{n}=396,947$ controls with no missing data available for complete cases analysis).

Household income was not included in the risk model as it was not necessary to include two variables measuring socio-economic deprivation and missing data were greater for household income. Income only reflects one aspect of deprivation, whereas the TDI takes into account income as well as car ownership, education, employment and number of persons per household. TDI is measured over previously-defined Output Areas, which contain $\sim 125$ households (30). Multivariate logistic regression analysis was then used to develop the final model (35) with model coefficient and odds ratio (OR) presented, with $95 \%$ confidence intervals.

Model performance was assessed by external validation using the North West Cohort from the UK Biobank, according to discrimination and calibration. Discrimination refers to the model's ability to separate those who develop the disease from those who do not. This is assessed using the C-statistic/area under the receiver operating curve (36). A value of 1 indicates perfect discrimination. Calibration compares observed and expected risks and was assessed using a calibration plot with points lying close to the 45 -degree line indicating good calibration (37). Bootstrapping was not completed as the dataset is sufficiently large, with minimal risk of optimism in predictions due to a good number of events per variable $(26,38,39)$.

\section{Results}

Descriptive statistics. Characteristics of all cases and controls are shown in Table I and differences are summarised below. Males accounted for $62 \%$ of cases $(n=165)$ compared with $45.4 \%(n=191,897)$ of controls $(\mathrm{P}<0.001)$. Patients with HNC were older at recruitment compared with controls [males 58 years (range, $41-70$ years) vs. 56 years (range, 37-72 years); females 59 years (range, $40-70$ years) vs. 56 years (39-71 years); $\mathrm{P}<0.001$ both sexes). Male cases were less likely to report 'never smoking' compared with male controls [32.9\% $(n=143)$ of male cases were never smokers vs. $49.2 \%(n=93,885)$ of male controls $(\mathrm{P}<0.001)]$. Amongst females, $45.9 \%(\mathrm{n}=119)$ of cases are never smokers vs. $60 \%(\mathrm{n}=137,805)$ of controls $(\mathrm{P}<0.001)$. More detailed measures of smoking exposure were analysed; 


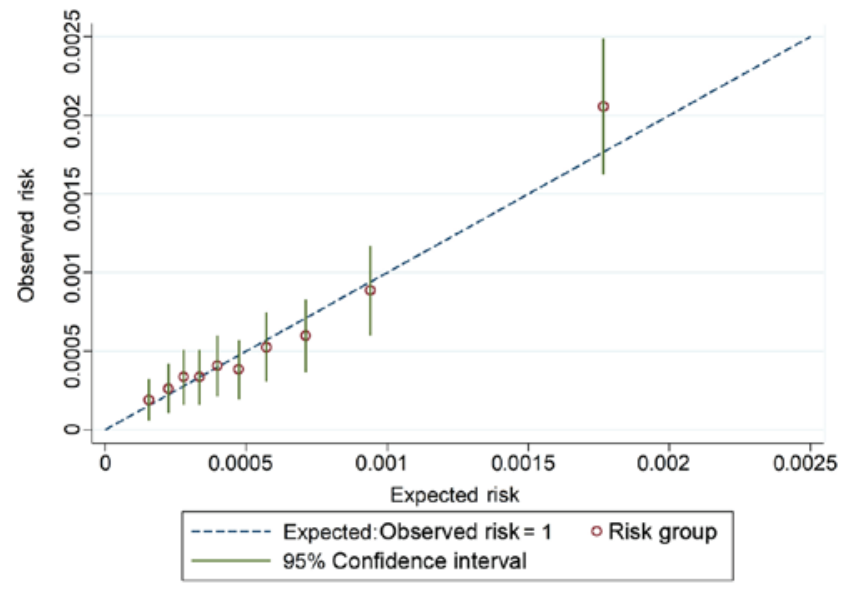

Figure 1. Calibration plot for the multivariate model for prediction of head and neck cancer. Expected risk is plotted against observed risk. Perfect calibration is indicated by the 45 -degree line.

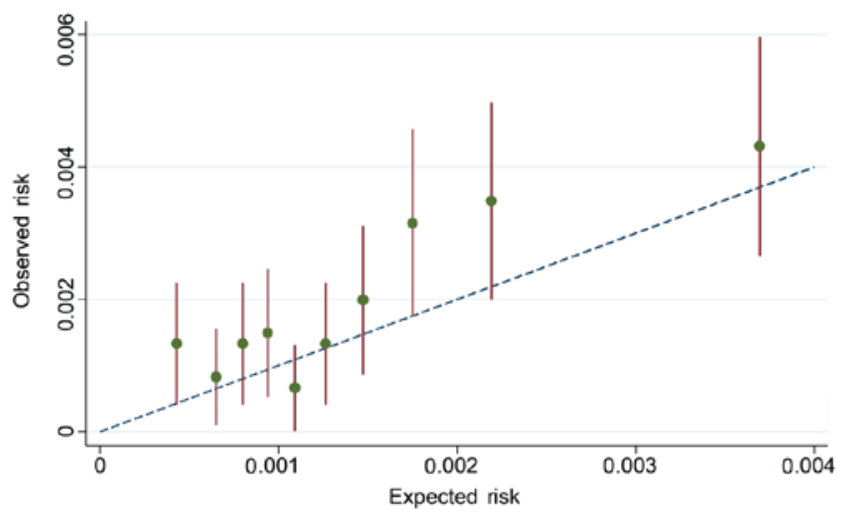

Expected: Observed risk=1 $1-95 \%$ Confidence interval - $\quad$ Risk group

Figure 2. Calibration plot for validation of the head and neck cancer prediction model using external data. Expected risk is plotted against observed risk. Perfect calibration is indicated by the 45 -degree line.

however, for smoking duration, the amount of missing data was high $(15.9 \%)$, therefore this was excluded from the risk model.

Male and female cases were significantly more likely to report daily consumption of alcohol compared with controls [30.9\% ( $n=136)$ of male cases vs. $25.8 \%(n=49,388)$ of male controls; $\mathrm{P}<0.001 ; 18.3 \%(\mathrm{n}=48)$ of female cases vs. $16.5 \%$ $(n=38,101)$ of female controls; $\mathrm{P}<0.001]$. Further detail on consumption of different types of alcohol can be found in Table SI. Significantly more male and female cases reported drinking 'less alcohol now compared to 10 years previously' ( $\mathrm{P}<0.017$ and $<0.001$ for males) (data not shown).

Male and female cases had significantly lower BMI compared with controls [mean 26.4 vs. 27.8 for males $(\mathrm{P}<0.001)$; 26.1 vs. 27.0 for females $(\mathrm{P}=0.004)$, respectively]. Fruit and vegetable consumption was lower amongst male and female cases compared with controls; $29.8 \%(n=131)$ of male cases consumed $<5$ pieces of fruit and vegetables per day vs. $20.7 \%(n=39,648)$ of controls; $P<0.001$. The figures for female cases are $19.1 \%(n=50)$ vs. $13.3 \% n=30,843$ in controls $(\mathrm{P}=0.006)$. In total, $31.6 \%(\mathrm{n}=139)$ of male cases were in most-socioeconomically deprived groups (Townsend IV and V) vs. $22.8 \%$ of controls $(\mathrm{n}=43,572 ; \mathrm{P}<0.001)$. In addition, $19.5 \%$ of males $(n=80)$ reported 0 days of exercise per week vs. $13 \%$ of controls ( $\mathrm{n}=28,823 ; \mathrm{P}<0.001)$.

Univariate analysis. The results of the univariate (unadjusted) analysis are shown in Table II. Increasing age, male sex, current or previous smoking, current and previous alcohol consumption and residence in an area of deprivation (TDI 4 or 5) were associated with increased risk of HNC. Eating greater $\geq$ five pieces of fruit and vegetables per day and partaking in moderate exercise at least once per week was associated with a protective effect against HNC. A household income of $£ 31,000-100,000$ per annum (compared with $<£ 18,000$ ) was also protective. Consuming alcohol only one to three times monthly or only on special occasions was significantly protective against $\mathrm{HNC}$, when compared with daily drinking.

Data on number of sexual partners was missing in $21 \%$ $(n=64)$ of cases (data not shown) and there was no significant difference in mean lifetime number of sexual partners on univariable analysis $(\mathrm{OR}=1.00 ; 95 \%$ CI 0.99-1.00; $\mathrm{P}=0.656)$, therefore this variable was excluded from the model.

Multivariate model. Table III shows the ORs for predictors in the multivariate model. Older age and male sex were significantly associated with increased risk of $\mathrm{HNC}$, with $4 \%$ increased risk with every increasing year of age $(\mathrm{OR}=1.04$; 95\% CI, 1.02-1.05). Males had nearly twice the risk of HNC compared with females $(\mathrm{OR}=1.81 ; 95 \% \mathrm{CI}, 1.38-2.38)$. Living in an area of social deprivation (TDI group 4 or 5) was associated with an increased risk of $\mathrm{HNC}(\mathrm{OR}=1.81$; 95\% CI 1.25-2.63 for TDI group 4). Eating at least five portions of fruit or vegetables per day offered a significant protective effect against $\mathrm{HNC}(\mathrm{OR}=0.71$; 95\% CI, 0.53-0.97), as did partaking in moderate exercise at least once per week $(\mathrm{OR}=0.68 ; 95 \% \mathrm{CI}$, 0.47-0.98) and five or more times per week $(\mathrm{OR}=0.66 ; 95 \% \mathrm{CI}$, 0.46-0.96). Higher BMI also conferred a protective effect $(\mathrm{OR}=0.96$; 95\% CI, 0.93-0.99).

Model performance. Discrimination, as measured by the C-statistic, was 0.69 (95\% CI, 0.66-0. 71), which demonstrated that the model was better compared with chance at predicting a case of HNC. The calibration plot is shown in Fig. 1. Expected and observed risk are plotted for ten risk groups, and the model displays good calibration. The 45-degree line indicates perfect calibration. CIs are narrow, due to the large number of participants. Most of the deciles are clustered close to the left side of the graph, indicating the very low risk of head and neck cancer in the general population.

External validation in the North West cohort. Characteristics of cases and controls within the validation dataset are presented in Table I. The prevalence of HNC in this population was 198 per 100,000 compared with 165 per 100,000 in the development dataset, indicating the higher risk of disease in this population. In total, 60,240 individuals (76.4\%) were available for complete case analysis (contained no missing data). The C-statistic for the model in the validation data is 0.64 (95\% CI, 0.60-0.68) (Table III), which shows that the 
model is better than chance at predicting the outcome. This value was close to the $\mathrm{C}$-statistic obtained from apparent discrimination indicating the performance was similar in the validation dataset. The risk of disease was very low and the observed and expected probabilities are close to the reference line, demonstrating reasonable calibration. The calibration slope was 0.83 and the calibration plot (Fig. 2) showed several data points lying above the reference line, which demonstrated that the model slightly under-predicted risk of head and neck cancer.

\section{Discussion}

The present study developed and validated of a risk prediction model for HNC using the UK Biobank dataset, with the aim of highlighting the potential for the use of risk prediction models within HNC. This risk model allows the calculation of an individual risk score for development of HNC.

The model performance was good in terms of its ability to discriminate between cases and controls $(\mathrm{C}$-statistic $=0.69)$ and displayed good calibration. The model was validated in a sub-group of individuals from the North West of England, known to have a higher incidence of HNC (2). The performance of the model in this external validation was reasonable, with a C-statistic of 0.64 and a calibration slope of 0.67 . Further validation should be performed using a truly independent dataset, to confirm the transportability of the present model to other populations. This C-statistic indicates good discrimination of the model but there is potential for improvement. For example, Lee et al reported area under the curve values (compared with the C-statistic) of 0.701-0.798 for a risk prediction models for oral and oropharyngeal cancer for men and women in the US population (40). The better performance compared with the present study may be attributed to the much larger sample size in their cohort.

Risk prediction modelling has been identified as an area of opportunity for early detection of cancer by the National Cancer Institute (41). The power of big data is increasingly recognised (42), and analysis of large population databases allows predictions to be made regarding the development of specific diseases in individuals, based on demographics and risk behaviours (42). Risk models have been shown to improve selection criteria for lung cancer screening programmes (12), for example the Liverpool Lung Project risk model (10) is currently used to guide recruitment of individuals at a high-risk of lung cancer to the UK Lung Cancer Screening trial (43). This demonstrates that with proper development, validation and implementation, risk models can be of benefit to both physicians and patients for numerous diseases, including HNC.

Increasing age, male sex, smoking and alcohol consumption are well established risk factors for HNC $(20,44,45)$, and these were confirmed by the present study. Previous alcohol consumption was identified as a risk factor for $\mathrm{HNC}$ in the present study. Alcohol is a recognised risk factor for $\mathrm{HNC}$, particularly when combined with either current or previous history of smoking $(18,19)$. The present study reveals previous consumption of alcohol appears to be a greater risk factor for HNC compared with current drinking. It is possible that those currently not drinking have stopped consuming alcohol for health-related reasons, for example alcoholic liver disease. The UK Biobank does not contain data regarding lifetime alcohol consumption, therefore this is a limitation of the present study. However, a significantly greater number of male and female cases reported drinking 'less alcohol now than 10 years previously' compared with controls (see results), which suggested a pattern of previous higher alcohol consumption amongst $\mathrm{HNC}$ cases.

Consuming at least five portions of fruit and vegetables (combined) per day is protective against HNC. Chuang et al have also published evidence of the protective effect of fruit and vegetables on the risk of HNC (46). Their study used the international INHANCE (International Head and Neck Cancer Epidemiology) consortium of studies with 14,520 cases and 22,737 controls; Consuming fruit 7 or more times per week offered a protective effect of $48 \%$ [OR 0.52 (95\% CI, 0.43-0.62)] and vegetables OR 0.66 (95\% CI, 0.49-0.90). This may be due to the antioxidant effects of fruits and vegetables as reactive oxygen species (ROS)-mediated DNA-damage is known to be a significant factor in carcinogenesis (47). Antioxidants are involved in the prevention of cellular and tissue damage caused by ROS and therefore have a well-established role in cancer prevention (48).

BMI has been investigated in association with HNC risk, with mixed results (49). The present study reported that increasing BMI confers a protective effect against HNC. A pooled analysis of 17 international studies also appeared to show a protective effect of higher BMI against HNC amongst smokers and consumers of alcohol (BMI >30; $\mathrm{OR}=0.38 ; 95 \% \mathrm{CI}, 0.30-0.49)$; however, this protective effect was not significant for never smokers (OR=0.95; 95\% CI, 0.47-1.91) (40). A similar tendency for leanness (BMI <25) has been noted in other smoking-related malignancies, such as lung and oesophageal cancer (50). The reason for this is unclear; however, this could be explained by residual confounding by smoking or another risk factor, reverse causation or a true effect. Smokers with lower BMI have been found to have higher levels of 8-hydroxydeoxyguanosin, a marker of oxidative DNA damage (51), which is associated with increased risk of various cancer types, including breast, lung, prostate and bladder cancer (52).

Hashibe et al investigated risk factors for $\mathrm{HNC}$ within prostate, lung, colorectal and ovarian cancer $(n=101,182)$ and demonstrated that physical activity for $>3 \mathrm{~h}$ per week offered a protective effect against $\mathrm{HNC}$ of $\sim 40 \%$ (OR=0.58; 95\% CI, $0.35-0.96)$ (20). The present study also reported that moderate exercise, for at least ten minutes, at least once per week, offers a protective effect against HNC. Current NHS guidelines recommend moderate exercise for $150 \mathrm{~min}$ per week and strength exercises on $\geq 2$ days per week. The guidance suggests that one way to do the recommended $150 \mathrm{~min}$ of weekly physical activity is to do 30 min on 5 days every week (32).

This study has confirmed a role for social deprivation in HNC, with those living in deprived areas (TDI 4) having an increased risk of developing HNC compared with those people living in the most affluent areas (TDI 1). This supports a previous study which demonstrates $\mathrm{HNC}$ is more common in lower socio-economic groups and those with lower educational attainment compared with those in higher socio-economic groups (OR=1.9; 95\% CI, 1.6-2.3) (53). This highlights the 
issue of social inequality in $\mathrm{HNC}$ that has been identified by the International Association for Cancer Research as a research priority (54).

It was not possible to separate $\mathrm{HNC}$ cases associated with HPV status, as this information is not captured by ICD-10 codes for head and neck cancer types. A future model for risk of HPV-associated HNC should be considered, if a suitable dataset were to become available. The present model is intended for use in primary care and it is unlikely that a patient's HPV status would be available for entry into a risk calculator, therefore HPV-status would not be a helpful addition to the risk prediction model in this context (40).

A limitation of the present study was that data regarding family history of HNC was not available from the UK Biobank, therefore this could not be considered as a potentially relevant risk factor. In addition, the majority of participants within the UK Biobank were born in the UK or Republic of Ireland (93.3\% of cases and $92.6 \%$ of controls), which may limit the application of the present model outside to these populations. The model could be externally validated in other populations to test its transportability, if a suitable database were available.

General dental practitioners are required to screen all patients for oral cancer (55). Using a personalised risk estimate could identify high-risk patients and support discussions between dental professionals and patients regarding risk behaviours, such as smoking and alcohol consumption. This would also provide the opportunity to discuss health promoting behaviours, such as eating fresh fruit and vegetables and taking regular exercise. There is evidence from a randomized controlled trial that use of a risk score when providing smoking cessation advice results in longer term success with smoking cessation (6).

In future, a web-based tool could be co-designed by patients and clinicians to allow easy generation of the risk score. Feasibility and clinical utility studies would be required to test whether the present $\mathrm{HNC}$ risk model is well-received by clinicians and patients. An impact study could then be completed to assess the effect on smoking cessation rates and other risk behaviours.

To the best of our knowledge, the present study was the first to develop and validate a risk model for head and neck cancer in the UK population, using statistical methodology developed according to TRIPOD guidelines (13). The model provides the opportunity to calculate a risk score, which can be used to discuss personalised risk of HNC with patients. This foundation could be built upon by including salivary biomarkers $(56,57)$ such as loss of heterozygosity at $9 p, 17 p$ and $4 \mathrm{q}(58)$, in future iterations of the model. Risk prediction modelling is currently under-utilised in HNC research; there is great potential to build, validate and implement risk calculators in several areas of HNC clinical practice. The model developed should be further assessed for clinical utility particularly for its suitability to screen individuals at high-risk of HNC and recruit them to clinical trials, as well as to guide dental practitioners when counselling patients on risk behaviours.

\section{Acknowledgements}

The authors would like to thank Professor Stephen Duffy (Centre for Cancer Prevention, Queen Mary University of
London) for his invaluable advice regarding the methodology for this study.

\section{Funding}

Laura Bonnett was funded by a National Institute for Health Research (NIHR) Post-Doctoral Fellowship (grant no. PDF-2015-08-044) for this research project. Caroline McCarthy was funded by a NIHR Academic Clinical Fellowship in Oral Medicine.

\section{Availability of data and materials}

The data that support the findings of this study are available on application from the UK Biobank but restrictions apply to the availability of these data, which were used under license for the current study, and so are not publicly available.

\section{Authors' contributions}

JKF and MWM developed the concept for the head and neck cancer risk prediction model. CEM undertook the preparation of the UK Biobank dataset for the analysis, discussed risk parameters with patients with head and neck cancer and those at risk, completed the statistical analysis and drafted the manuscript. LJB and MWM designed the methodology, supported the statistical analysis and assisted with manuscript editing. JKF helped critically revise the manuscript for important intellectual content and supervised the analysis. All authors read and approved the final manuscript.

\section{Ethics approval and consent to participate}

The Research Ethics Committee reference for UK Biobank is 16/NW/0274.

\section{Patient consent for publication}

Not applicable.

\section{Competing interests}

The authors declare that they have no competing interests.

\section{References}

1. Smittenaar CR, Petersen KA, Stewart K and Moitt N: Cancer incidence and mortality projections in the UK until 2035. Br J Cancer 115: 1147-1155, 2016.

2. Taib BG, Oakley J, Dailey Y, Hodge I, Wright P, du Plessis R, Rylands J, Taylor-Robinson D, Povall S, Schache A, et al: Socioeconomic deprivation and the burden of head and neck cancer-regional variations of incidence and mortality in merseyside and cheshire, North West, England. Clin Otolaryngol 43: 846-853, 2018.

3. Brocklehurst P, Kujan O, O'Malley LA, Ogden G, Shepherd S and Glenny AM: Screening programmes for the early detection and prevention of oral cancer. Cochrane Database Syst Rev 19: CD004150, 2013.

4. van der Waal I: Are we able to reduce the mortality and morbidity of oral cancer; some considerations. Med Oral Patol Oral Cir Bucal 18: e33-e37, 2013. 
5. Friedrich RE: Delay in diagnosis and referral patterns of 646 patients with oral and maxillofacial cancer: A report from a single institution in Hamburg, Germany. Anticancer Res 30: $1833-1836,2010$

6. Sherratt FC, Marcus MW, Robinson J and Field JK: Utilizing lung cancer risk prediction models to promote smoking cessation: Two randomized controlled trials. Am J Health Promot 32: $1196-1205,2018$

7. World Health Organisation. Guide to cancer early diagnosis, 2017.

8. Pfeiffer RM, Park Y, Kreimer AR, Lacey JV Jr, Pee D, Greenlee RT, Buys SS, Hollenbeck A, Rosner B, Gail MH and Hartge P: Risk prediction for breast, endometrial, and ovarian cancer in white women aged 50 year or older: Derivation and validation from population-based cohort studies. PLoS Med 10: e1001492, 2013.

9. Tammemagi CM, Pinsky PF, Caporaso NE, Kvale PA, Hocking WG, Church TR, Riley TL, Commins J, Oken MM, Berg CD and Prorok PC: Lung cancer risk prediction: Prostate lung, colorectal and ovarian cancer screening trial models and validation. J Natl Cancer Inst 103: 1058-1068, 2011.

10. Marcus MW, Chen Y, Raji OY, Duffy SW and Field JK: LLPi Liverpool lung project risk prediction model for lung cancer incidence. Cancer Prev Res (Phila) 8: 570-575, 2015.

11. Ten Haaf K, Jeon J, Tammemägi MC, Han SS, Kong CY Plevritis SK, Feuer EJ, de Koning HJ, Steyerberg EW and Meza R: Risk prediction models for selection of lung cancer screening candidates: A retrospective validation study. PLoS Med 14: e1002277, 2017.

12. Tammemagi MC, Schmidt H, Martel S, McWilliams A, Goffin JR, Johnston MR, Nicholas G, Tremblay A, Bhatia R, Liu $\mathrm{G}$ et al: Participant selection for lung cancer screening by risk modelling [the Pan-Canadian Early Detection of Lung Cancer (PanCan) study]: A single-arm, prospective study. Lancet Oncol 18: 1523-1531, 2017.

13. Collins GS, Reitsma JB, Altman DG and Moons KG: Transparent reporting of a multivariable prediction model for individual prognosis or diagnosis (TRIPOD): The TRIPOD statement. Br J Cancer 112: 251-259, 2015

14. UK Biobank: Protocol for a large-scale prospective epidemiological resource, 2007.

15. Public Health England (PHE): National Cancer Registration and AnalysisService.https://www.gov.uk/guidance/national-cancer-registration-and-analysis-service-ncras. Accessed November 3, 2016.

16. World Health Organisation (WHO): International statistical classification of diseases and related health problems, 10th revision (ICD-10). Vol 2. 5th edition. WHO, Geneva, 2016.

17. Hashim D, Genden E, Posner M, Hashibe M and Boffetta P: Head and neck cancer prevention: From primary prevention to impact of clinicians on reducing burden. Ann Oncol 30: 744-756, 2019.

18. Hashibe M, Brennan P, Benhamou S, Castellsague X, Chen C, Curado MP, Dal Maso L, Daudt AW, Fabianova E, Fernandez L, et al: Alcohol drinking in never users of tobacco, cigarette smoking in never drinkers, and the risk of head and neck cancer: Pooled analysis in the international head and neck cancer epidemiology consortium. J Natl Cancer Inst 99: 777-789, 2007.

19. Hashibe M, Brennan P, Chuang SC, Boccia S, Castellsague X, Chen C, Curado MP, Dal Maso L, Daudt AW, Fabianova E, et al: Interaction between tobacco and alcohol use and the risk of head and neck cancer: Pooled analysis in the international head and neck cancer epidemiology consortium. Cancer Epidemiol Biomarkers Prev 18: 541-550, 2009.

20. Hashibe M, Hunt J, Wei M, Buys S, Gren L and Lee YC: Tobacco, alcohol, body mass index, physical activity, and the risk of head and neck cancer in the prostate, lung, colorectal, and ovarian (PLCO) cohort. Head Neck 35: 914-922, 2013.

21. Shaw R and Beasley N: Aetiology and risk factors for head and neck cancer: United Kingdom national multidisciplinary guidelines. J Laryngol Otol 130 (Suppl 2): S9-S12, 2016

22. D'Souza G, McNeel TS and Fakhry C: Understanding personal risk of oropharyngeal cancer: Risk-groups for oncogenic oral HPV infection and oropharyngeal cancer. Ann Oncol 28 : 3065-3069, 2017

23. Heck JE, Berthiller J, Vaccarella S, Winn DM, Smith EM, Shan'gina O, Schwartz SM, Purdue MP, Pilarska A, Eluf-Neto J, et al: Sexual behaviours and the risk of head and neck cancers: A pooled analysis in the international head and neck cancer epidemiology (INHANCE) consortium. Int J Epidemiol 39: 166-181, 2010
24. Heus P, Damen JAAG, Pajouheshnia R, Scholten RJPM, Reitsma JB, Collins GS, Altman DG, Moons KGM and Hooft L: Poor reporting of multivariable prediction model studies: Towards a targeted implementation strategy of the TRIPOD statement. BMC Med 16: 120, 2018.

25. Moons KG, Kengne AP, Grobbee DE, Royston P, Vergouwe Y, Altman DG and Woodward M: Risk prediction models: II. External validation, model updating, and impact assessment. Heart 98: 691-698, 2012.

26. Riley RD, Ensor J, Snell KI, Debray TP, Altman DG, Moons KG and Collins GS: External validation of clinical prediction models using big datasets from e-health records or IPD meta-analysis: Opportunities and challenges. BMJ 353: i3140, 2016.

27. McCarthy CE, Field JK, Rajlawat BP, Field AE and Marcus MW: Trends and regional variation in the incidence of head and neck cancers in England: 2002 to 2011. Int J Oncol 47: 204-210, 2015.

28. Steyerberg EW, Vickers AJ, Cook NR, Gerds T, Gonen M, Obuchowski N, Pencina MJ and Kattan MW: Assessing the performance of prediction models: A framework for traditional and novel measures. Epidemiology 21: 128-138, 2010.

29. Collins GS, Ogundimu EO, Cook JA, Manach YL and Altman DG: Quantifying the impact of different approaches for handling continuous predictors on the performance of a prognostic model. Stat Med 35: 4124-4135, 2016

30. Townsend P: Deprivation. J Soc Policy 16: 125-146, 1987.

31. (NHS): Why 5 a day?, 2018. https://www.nhs.uk/live-well/ eat-well/why-5-a-day/. Accessed October 8, 2018

32. NHS: Physical activity guidelines for older adults, 2018 https://www.nhs.uk/live-well/exercise/physical-activity-guidelin es-older-adults/. Accessed October 8, 2019.

33. Little RJB and Rubin DB: Statistical analysis with missing data. 2nd edition, John Wiley \& Sons, Inc., Hoboken, 2014.

34. Heinze G, Wallisch C and Dunkler D: Variable selection-A review and recommendations for the practicing statistician. Biom J 60: 431-449, 2018

35. Hosmer DW and Lemeshow S: Applied logistic regression. John Wiley \& Sons, Inc., New York, NY, 1989.

36. Steyerberg EW, Harrell FE Jr, Borsboom GJ, Eijkemans MJ, Vergouwe Y and Habbema JD: Internal validation of predictive models: Efficiency of some procedures for logistic regression analysis. J Clin Epidemiol 54: 774-781, 2001.

37. Van Calster B, Nieboer D, Vergouwe Y, De Cock B, Pencina MJ and Steyerberg EW: A calibration hierarchy for risk models was defined: From utopia to empirical data. J Clin Epidemiol 74: 167-176, 2016.

38. Smith GC, Seaman SR, Wood AM, Royston P and White IR Correcting for optimistic prediction in small data sets. Am J Epidemiol 180: 318-324, 2014.

39. van der Ploeg T, Austin PC and Steyerberg EW: Modern modelling techniques are data hungry: A simulation study for predicting dichotomous endpoints. BMC Med Res Methodol 14: 137, 2014.

40. Lee YA, Al-Temimi M, Ying J, Muscat J, Olshan AF, Zevallos JP, Winn DM, Li G, Sturgis EM, Morgenstern H, et al: Head and neck cancer risk prediction models for the US population from the INHANCE consortium. Am J Epidemiol 189: 330, 2020.

41. Gail MH, Brinton LA, Byar DP, Corle DK, Green SB, Schairer C and Mulvihill JJ: Projecting individualized probabilities of developing breast cancer for white females who are being examined annually. J Natl Cancer Inst 81: 1879-1886, 1989.

42. Chawla NV and Davis DA: Bringing big data to personalized healthcare: A patient-centered framework. J Gen Intern Med 28 (Suppl 3): S660-S665, 2013

43. Field JK, Duffy SW, Baldwin DR, Brain KE, Devaraj A, Eisen T, Green BA, Holemans JA, Kavanagh T, Kerr KM, et al: The UK lung cancer screening trial: A pilot randomised controlled trial of low-dose computed tomography screening for the early detection of lung cancer. Health Technol Assess 20: 1-146, 2016.

44. Winn DM, Lee YC, Hashibe M and Boffetta P; INHANCE consortium: The INHANCE consortium: Toward a better understanding of the causes and mechanisms of head and neck cancer. Oral Dis 21: 685-693, 2015

45. Beynon RA, Lang S, Schimansky S, Penfold CM, Waylen A, Thomas SJ, Pawlita M, Waterboer T, Martin RM, May M and Ness AR: Tobacco smoking and alcohol drinking at diagnosis of head and neck cancer and all-cause mortality: Results from head and neck 5000, a prospective observational cohort of people with head and neck cancer. Int J Cancer 143: 1114-1127, 2018.

46. Chuang SC, Jenab M, Heck JE, Bosetti C, Talamini R, Matsuo K, Castellsague X, Franceschi S, Herrero R, Winn DM, et al: Diet and the risk of head and neck cancer: A pooled analysis in the INHANCE consortium. Cancer Causes Control 23: 69-88, 2012. 
47. Ziech D, Franco R, Pappa A and Panayiotidis MI: Reactive oxygen species (ROS)-induced genetic and epigenetic alterations in human carcinogenesis. Mutat Res 711: 167-173, 2011.

48. Choudhari SK, Chaudhary M, Gadbail AR, Sharma A and Tekade S: Oxidative and antioxidative mechanisms in oral cancer and precancer: A review. Oral Oncol 50: 10-18, 2014.

49. Gaudet MM, Olshan AF, Chuang SC, Berthiller J, Zhang ZF, Lissowska J, Zaridze D, Winn DM, Wei Q, Talamini R, et al: Body mass index and risk of head and neck cancer in a pooled analysis of case-control studies in the international head and neck cancer epidemiology (INHANCE) consortium. Int J Epidemiol 39: 1091-1102, 2010.

50. Bhaskaran K, Douglas I, Forbes H, dos-Santos-Silva I, Leon DA and Smeeth L: Body-mass index and risk of 22 specific cancers: A population-based cohort study of 5.24 million UK adults. Lancet 384: 755-765, 2014.

51. Mizoue T, Kasai H, Kubo T and Tokunaga S: Leanness, smoking, and enhanced oxidative DNA damage. Cancer Epidemio Biomarkers Prev 15: 582-585, 2006.

52. Wu LL, Chiou CC, Chang PY and Wu JT: Urinary 8-OHdG: A marker of oxidative stress to DNA and a risk factor for cancer, atherosclerosis and diabetics. Clin Chim Acta 339: 1-9, 2004.
53. Radoi L and Luce D: A review of risk factors for oral cavity cancer: The importance of a standardized case definition. Community Dent Oral Epidemiol 41: 97-109, e78-e91, 2013.

54. International Agency for Research on Cancer (IARC): Reducing social inequalities in cancer: Evidence and priorities for research. IARC Scientific Publication No. 168. Vaccarella S, Lortet-Tieulent J, Saracci R, Conway DI, Straif K and Wild CP (eds). IARC, Lyon, France, 2019.

55. Faculty of general dental practice (UK): Standards in Dentistry, 2018.

56. Achalli S, Madi M, Babu SG, Shetty SR, Kumari S and Bhat S: Sialic acid as a biomarker of oral potentially malignant disorders and oral cancer. Indian J Dent Res 28: 395-399, 2017.

57. Kallalli BN, Rawson K, Muzammil, Singh A, Awati MA and Shivhare P: Lactate dehydrogenase as a biomarker in oral cancer and oral submucous fibrosis. J Oral Pathol Med 45: 687-690, 2016.

58. Rock LD, Rosin MP, Zhang L, Chan B, Shariati B and Laronde DM: Characterization of epithelial oral dysplasia in non-smokers: First steps towards precision medicine. Oral Oncol 78: 119-125, 2018. 